
internationales

vol. 30 - n² | 2014

Composer (avec) la frontière. Passages, parcours migratoires et échanges sociaux

\title{
Parcours dans un paysage flottant de frontières
}

\author{
A Journey in a Floating Borderland
}

Un viaje en un paisaje flotante de fronteras

\section{Michel Agier}

\section{(2) OpenEdition}

\section{Journals}

\section{Édition électronique}

URL : https://journals.openedition.org/remi/6845

DOI : $10.4000 /$ remi.6845

ISSN : $1777-5418$

\section{Éditeur}

Université de Poitiers

\section{Édition imprimée}

Date de publication : 1 juin 2014

Pagination : 13-23

ISBN : 979-10-90426-22-1

ISSN : 0765-0752

\section{Référence électronique}

Michel Agier, "Parcours dans un paysage flottant de frontières », Revue européenne des migrations internationales [En ligne], vol. 30 - n² | 2014, mis en ligne le 01 juin 2017, consulté le 15 février 2023 URL : http://journals.openedition.org/remi/6845; DOI : https://doi.org/10.4000/remi.6845 


\section{Parcours dans un paysage flottant de frontières}

\section{Michel Agier ${ }^{1}$}

Je m'interrogerai dans cet article sur ce que peut être, du point de vue des terrains ethnographiques, un paysage de frontières, ou sur le fait de savoir en quoi les frontières peuvent finir par former des paysages. Je préciserai d'abord le sens des deux principaux termes de cette proposition : paysage et frontière. Avec le " paysage ", je cherche à inclure les observations localisées dans un ensemble plus vaste sans réduire la force de chaque situation observée. Le terme est devenu familier aux anthropologues après qu'Arjun Appadurai I'ait utilisé pour conceptualiser la perception de réseaux, de flux ou de "nébuleuses " existant à l'échelle globale, au-delà des cadres locaux. Selon lui, le terme de " paysage ", dans un usage métaphorique (-scape), aurait défini idéalement les mondes des capitaux, du progrès technologique et industriel, des communications, des idées ou des groupes humains, tous largement délocalisés (Appadurai, 2005). Mais l'intérêt de la notion a été d'emblée réduit par le manque d'ancrage de l'analyse, opposant de manière trop systématique le "local " et le " global " et faisant de ce dernier terme une réalité en soi, autonome. Cette opposition s'est avérée finalement très idéologique et politique comme l'ont montré les sociologues de la mondialisation Zygmunt Bauman (1999) ou Jean-François Bayart (2004), selon qui les "globaux " forment une classe sociale supérieure (auto-)perçue comme globale, excessivement visible ou puissante, distincte, voire opposée aux "locaux".

\section{Borderland. La frontière comme paysage}

La notion de paysage n'en reste pas moins une bonne entrée en matière pour dépasser le cadre immédiat de la chose observée (lieu, situation, groupe) et de proche en proche tenter de saisir les ramifications jusqu'à la dimension mondialisée, planétaire de certains phénomènes. Pour autant, il est essentiel de pouvoir y raccrocher la texture et la " rugosité " du monde (Agier, 2014b), ce que I'on ne saisira que par l'enquête locale. II s'agit donc de décrire des objets empiriques locaux dont la globalité peut être appréhendée sans renoncer en rien au local ; en d'autres termes, de déployer une ethnographie globale pour reprendre

1 Anthropologue, Directeur de recherche IRD, Directeur d'études à I'EHESS, CEAf (IRD -

EHESS), 96 boulevard Raspail, 75006 Paris ; michel.agier@ehess.fr 
les termes de Michael Burawoy $(2000)^{2}$. Une des manières de faire consisterait idéalement en des enquêtes collectives à la fois multilocales et simultanées. À défaut de pouvoir mettre en œuvre cette simultanéité et cette ubiquité - qui caractérisent d'ailleurs la perception de la globalisation du monde - tout en restant en même temps au plus près du terrain comme le veut la recherche anthropologique, l'on peut considérer que la contemporanéité et la multilocalité $d^{\prime}$ 'une question ou d'un phénomène à l'échelle mondiale peuvent être la base et le point de départ d'un programme d'ethnographie globale.

$C^{\prime}$ est la question de la frontière qui réunit entre eux les différents lieux qu'on souhaite relier ici. Avec ce second terme associé à " paysage ", il s'agit de poursuivre et $d$ 'approfondir l'analyse des transformations de la frontière dans le monde contemporain, abordée antérieurement (Agier, 2013). La mondialisation n'a pas supprimé les frontières, elle les a au contraire multipliées, mais aussi déplacées, disséminées et modifiées. Au-delà des seules frontières administratives nationales ou des frontières urbaines identifiées comme telles par une limite visible et plus ou moins matérialisée, c'est donc l'épreuve des situations de frontière en général qui nous intéressera ici. Ce qui suppose que la frontière comme limite peut aussi être appréhendée du point de vue de son élargissement ou de son épaississement dans le temps et dans l'espace. Comme si les difficultés nouvelles associées au passage des frontières, à la résolution des conflits dans les situations de frontière, et à la violence parfois mortelle aux frontières, avaient un effet grossissant en montrant toute l'épaisseur temporelle et spatiale de la ligne frontalière elle-même.

Ce regard à la loupe montre aussi les microcosmes d'une mondialisation qui n'a rien d'enchantée, loin de là, ainsi qu'un cosmopolitisme ordinaire. Je distingue ce cosmopolitisme ordinaire de la "bulle globale " dans laquelle se trouvent les experts internationaux, technocrates, leaders et créateurs d'images qui parlent du monde, de la globalisation et se voient ou sont vus eux-mêmes comme cosmopolites, qui circulent vite, de manière fluide, d'un lieu à un autre de la planète, et n'ont pas besoin de beaucoup bouger pour se déplacer puisqu'ils ne se confrontent guère à la dureté des frontières ni à la longueur et pénibilité des déplacements. Le monde lui-même, avec toutes ses frontières, son relief, ses détritus, ses odeurs, compose plutôt le décor flouté de leur libre et cotonneux voyage.

L'ordinaire cosmopolite, lui, est l'expérience vécue, quotidienne et ordinaire, de la frontière, une expérience " ancrée " du partage du monde, aussi inégalitaire, conflictuelle ou violente soit cette expérience de partage. Cette épreuve est faite d'arrangements quotidiens de la part de celles et ceux qui sont dans le " labyrinthe de l'étranger " (Schütz, 2010) sans avoir encore réussi à en sortir, qui s'installent dans la situation de frontière, se confrontent avec difficulté aux autres langues, manières de faire et de penser, s'adaptent et se transforment par cet exercice obligé, mais qui doivent aussi faire avec la matérialité et la socialité inédites et " marginales " dans laquelle ils se trouvent.

Ce décentrement systématique et épistémologique dans la frontière nous fait porter le regard à la fois sur la frontière elle-même et sur le monde vu depuis la

2 Voir aussi Gupta et Ferguson (1997). 
frontière. De là, il est plus évident qu'une bonne part du malaise contemporain vient du fait qu'avec la mondialisation humaine, avec le développement des circulations dans des périmètres toujours plus vastes, des points plus éloignés et des mobilités plus fréquentes, les situations de frontière sont plus nombreuses, plus complexes, alors que les personnes engagées dans ces situations y sont peu ou pas préparées. Faute de les percevoir comme telles, nous risquons tous ensemble de perdre de vue l'importance voire la centralité qu'elles occupent dans la "fabrication " du monde, de ne pas voir leur potentialité d'apprentissage, de création, de transformation pour le monde en général.

C'est donc l'identification de la situation de frontière en ces lieux d'enquête qui nous servira de fil conducteur - une approche que le terme anglais borderland synthétise bien - un " paysage de frontières " donc, et cela dans deux sens. D'une part, chaque lieu évoqué représente lui-même un paysage de frontière, un microcosme où des personnes sont étrangères aux yeux de celles qui sont établies là, mais aussi relativement étrangères les unes aux autres. Elles se croisent et se rencontrent, échangent et négocient leurs places respectives, et créent ainsi un petit monde "banalement " cosmopolite. En même temps, ces lieux forment une limite par rapport à une centralité urbaine et politique, dont ils sont la marge, et ce rapport les détermine ${ }^{3}$. D'autre part, l'ensemble des lieux de frontière mis côte à côte représente un autre " paysage ", cette fois à l'échelle globale, planétaire. La possibilité de cet assemblage suppose de dépasser l'épistémologie comparatiste pour aller vers celle du "dispositif ", comme on l'a fait récemment, dans un projet collectif, à propos du dispositif des camps et campements dans le monde. Ce dispositif est à l'œuvre dans les circulations des personnes, des organisations et des savoirs (Agier, 2014a).

Mais avant de nous interroger sur ce qui fait un éventuel paysage continu à l'échelle mondiale, il convient d'établir la récurrence de ce phénomène qu'on a identifié comme le produit de l'extension dans le temps et dans l'espace des situations de frontière. Pour ce faire, je prendrai trois exemples de lieu des marges urbaines, dont chacun représente à sa manière une frontière - ce qui me permettra aussi de faire une lecture rétrospective de trois terrains d'enquête où je me suis trouvé confronté, dans des contextes locaux apparemment très différents (en Afrique, en Europe et au Proche-Orient), à la répétition des situations de frontière urbaine.

\section{Le zongo de Lomé, cinq fois à la marge}

Mes premières recherches ont porté sur les quartiers, dits zongo, dans une partie de l'Afrique de l'Ouest - entre Nigeria, Niger, Burkina Faso, Bénin, Togo et Ghana, c'est-à-dire sur les routes anciennes des commerçants itinérants haoussas. Ce sont des quartiers d'étrangers dès leur fondation, dans la deuxième moitié du XIXe siècle, jusqu'aujourd'hui. Le terme zongo (ou zango) est un mot de la langue haoussa (vaste groupe ethnique d'environ 20 millions de personnes, originaires du Nord-Nigeria et du Niger et connus en Afrique de

3 Sur le rapport entre le centre (incarné par la Ville ou par l'État) et ses marges et sur son inversion dans la " centralité des marges ", voir Daas et Poole (2004) ; Doraï et Puig (2012). 
I'Ouest pour leur rôle important, passé et présent, dans les réseaux marchands). Le terme désigne les constructions provisoires, les paillotes, situées à la marge d'une ville, par opposition au terme birni, la ville dans son enceinte. On trouve des zongos dans toute la partie orientale de l'Afrique occidentale, depuis le NordNigeria jusqu'à Accra, au Ghana. Le zongo de Lomé (la capitale du Togo) aurait été fondé comme premier campement haoussa de la capitale togolaise par un chef de caravane (mai dugu) venu de Salaga (place marchande ancienne dans I'actuel Ghana) dans les années 1870 ; c'étaient quelques paillotes au bord des rares maisons de commerce sur la plage. Au fil des ans, d'autres commerçants et migrants, puis d'autres étrangers musulmans venus des régions africaines septentrionales, et enfin des migrants arrivés du centre et du nord du Togo ont trouvé là une sorte de refuge - "allochtones " (selon une terminologie officielle ancienne en Afrique de l'Ouest, qui désigne les personnes nées hors de la localité de résidence). Venus des régions de savane, ils arrivaient dans une ville côtière, très anciennement marquée par la présence européenne, par la scolarisation française et par l'importance des religions catholique et païenne (culte des vodun). Malgré une forte intégration collective et individuelle des gens du zongo à la vie sociale et économique de la capitale togolaise, le quartier a toujours gardé, pour les Loméens, son identité des premiers temps, celle que l'évêque de Lomé, en 1928, qualifiait comme étant le lieu des " étrangers sans domicile fixe ou sans parenté ni connaissance dans la localité " (Cessou, 1928 : 55).

Situé sur un emplacement initialement marginal - mais devenu au fil des ans une partie du centre de la capitale -, le quartier était jugé insalubre, trop dense, peuplé d'étrangers sans titre foncier et peu fiables sur le plan politique pour le pouvoir en place. Le zongo a été détruit à la fin des années 1970 et " déguerpi " aux dernières limites de l'agglomération urbaine. Puis le " nouveau zongo " se recomposa petit à petit, dépassant de manière parfois illégale les limites matérielles qui lui avaient d'abord été imposées. II est devenu trente ans plus tard l'une des zones périphériques les plus dynamiques de Lomé, faisant le lien entre la ville et les circulants, les migrants et les commerçants - dont il est le repère et le quartier d'accueil - et la partie septentrionale du pays vers laquelle il est orienté.

Ainsi, depuis la fondation du premier "campement haoussa " à la fin du $\mathrm{XIXe}$ siècle, le zongo a toujours été repoussé à la marge du périmètre urbain (il a connu en tout cinq " déguerpissements "), mais il s'est recréé socialement à chaque réinstallation. Les résidents se désignent parfois comme Haoussas, mais l'ethnonyme est extensible puisque les originaires des différentes régions soudano-sahéliennes (qui relèvent près de cinquante identités ethniques différentes) peuvent aussi, selon les contextes, se dire ou être désignés "Haoussas ". Mais plus encore (parmi les jeunes en particulier), les habitants se désignent avec le nom de "Zongolais ", un " urbanyme " plutôt qu'un ethnonyme donc, faisant du nom du lieu le nom de reconnaissance et d'identification dans le cadre urbain ${ }^{4}$.

4 Voir Agier (1999) et pour saisir l'évolution du quartier zongo et de la position des " étrangers " à Lomé, voir Spire (2011). 
Ainsi, l'étranger des villes, que les " Haoussas " ou "Zongolais " incarnent dès leur plus lointaine raison d'être et de se déplacer, et dans leur disposition territoriale, apparaît ici dans toute son ambiguïté. II incarne une tension permanente entre l'itinérance et la sédentarité : ce sont des "étrangers " qui n'ont pas abandonné la liberté d'aller et de venir, pour le dire comme Georg Simmel (1984), mais ils n'ont pas poursuivi leur chemin. Et c'est en tant qu'étrangers " chez eux " dans le quartier des étrangers qu'ils deviennent " établis " dans la capitale togolaise, étrangers logeurs d'étrangers, et doubles des locaux.

Topographiquement, le zongo est situé sur l'exacte limite du périmètre urbain et cette position de frontière entre la ville et son dehors lui a permis de connaître un nouveau développement tout en maintenant sa position. Non pas une " identité " ethnique, elle-même plus changeante et diversifiée qu'avant, mais une situation, une position et une condition de frontière.

\section{Le campement des migrants de Patras, sur la frontière}

Plus récemment, j'ai mené avec Sara Prestianni (photographe et coordinatrice du réseau associatif Migreurop) une enquête sur les lieux de relative fixation des migrants circulant en Europe à la recherche d'une installation relativement stable. En attendant d'atteindre cette hypothétique stabilité, ils circulent et s'établissent provisoirement au plus près des frontières. C'est le cas du campement de Patras en Grèce, qui fournira le deuxième exemple de cette enquête. Ce campement, qui a reçu de 500 à 2000 occupants selon les moments, est situé à quelques dizaines de mètres du port de la ville, d'où partent des camions de marchandises pour I'Italie. Les migrants tentent de monter dans les camions pour passer, cachés à l'arrière des véhicules qui sont chargés au fond de la cale des navires ${ }^{5}$.

Quand un nouveau arrive, il va d'abord dans l'immeuble squatté juste à côté des baraques. Puis il regarde s'il y a une place pour lui dans les abris. Ou alors, s'ils sont plusieurs nouveaux arrivants, ils construisent une maison. Quand il est arrivé, Yassir, Afghan Hazara, a trouvé quelqu'un qui venait de la même ville que lui - la même ville au Pakistan, pas en Afghanistan. Cette personne l'a invité à venir dans sa chambre. Maintenant il rend I'hospitalité à un autre, arrivé quelques mois après lui, Afghan Hazara venu de la même ville du Pakistan : ils ne se connaissaient pas, mais ils connaissaient les mêmes gens, "alors, je lui ai dit : viens ici !".

Mahmoud est celui qu'on présente comme le "leader " du campement. II est travailleur social de formation, mais là, dans la migration, il circule entre Patras et Athènes. II tient une des deux petites boutiques du campement. II dit : "Patras, c'est une ville hors-la-loi ".

Paradoxe de ces hors-lieux, après douze ans d'existence, le campement de Patras est devenu un lieu repère, un point fixe sur les routes multiples, mais toutes semblables, de la migration. Patras est connu de tous ceux qui tentent

5 Les notes qui suivent sont tirées de Agier et Prestianni (2011). 
ces routes. Aussi connu que Zahedan (à la frontière entre I'Iran, le Pakistan et l'Afghanistan) ou Calais, au nord de la France. Ces lieux sont devenus, en partie, des carrefours cosmopolites : ce sont les étapes de parcours qui ont le monde comme échelle, des parcours toujours risqués, imprévisibles, qui mènent de I'Afghanistan (ou du Pakistan, ou de I'Iran) à l'Europe... mais l'exil peut changer de périmètre - tel l'exil africain, qui va vers l'Europe, mais aussi plus récemment, vers le Proche-Orient, l'Amérique et l'Asie lointaine.

Ces repères cosmopolites sont aussi très locaux et ils le sont doublement si I'on s'en tient à ce qu'on sait de Patras et Calais. D'une part, ils ont plusieurs années d'existence : une patine s'est faite, de nouveaux arrivants ont trouvé des habitats déjà là, déjà construits et " habités ", ils s'y sont insérés comme on s'insère dans un "lieu anthropologique "(Augé, 1992) qui a déjà un peu d'histoire (douze ans d'existence à Patras, sept ans à Calais), un peu de relations internes (les amitiés nouées et développées dans les moments de l'attente, mais aussi les tensions anciennes avec les Irakiens ou les Soudanais), et aussi un peu d'identité externe (c'est à Calais qu'on a donné le nom "jungle des Afghans ", comme c'est en Grèce qu'on a parlé du " campement de Patras »). D’autre part, ces lieux font partie de I'histoire des villes où ils sont nés : histoires de conflits, mais aussi de solidarités à Patras comme à Calais, que les soutiens donnés par les habitants et associations de ces villes aient été humanitaires, juridiques ou politiques.

Le campement de Patras doit ses douze années d'existence (de fin 1996 jusqu'à sa destruction par le feu et les bulldozers en juillet 2009) à la répétition d'une " tolérance " municipale, résultat d'un compromis entre la pression de certains riverains peu accueillants, celle des associations de défense des droits des étrangers et celle des migrants cherchant leur voie vers le port/frontière. Malgré l'important turnover des occupants, certains ont pu s'établir et rester jusqu'à deux ans dans le campement, ouvrant une boutique de restauration, de produits de première nécessité, ou occupant des positions de leadership.

Lieu stable sur des parcours incertains, errants, le campement de Patras, comme celui de Calais, est lui-même une extension de la large frontière où se trouvent les migrants en errance aux " portes " de l'Europe, sans réussir à la franchir entièrement ${ }^{6}$.

\section{Gaza Hospital (Sabra, Beyrouth), le squat entre le camp et la ville}

Le troisième exemple que je souhaite présenter concerne un immeuble squatté de onze étages, appelé "Gaza Hospital ", dans le quartier de Sabra à Beyrouth. Construit dans les années 1970 par I'OLP (Organisation de Libération de la Palestine), lorsqu'elle avait son siège à Beyrouth dans le quartier de Sabra, I'hôpital a été progressivement abandonné après I'éviction de I'OLP en 1982 et son installation à Tunis. Partiellement détruit en 1982 par l'armée israélienne lors de son invasion du quartier (qui a donné lieu au massacre de Sabra et Chatila), puis brûlé lors de la " guerre des camps " et la " guerre interne " entre

6 Sur cette situation, voir Clochard (2012). 
1986 et 1987, il a été abandonné par tout service médical à la fin de ce dernier événement ${ }^{7}$. Selon les récits recueillis en 2012, le squat de Gaza Hospital aurait été fondé en 1987 par trois femmes palestiniennes qui avaient fui les violences du camp voisin de Chatila et erraient dans les rues de Sabra avec leurs enfants à la recherche d'un abri. Elles entrèrent dans le bâtiment qui avait été entièrement déserté suite à un incendie qui avait endommagé plusieurs étages. L'armée syrienne occupant alors la zone de Sabra les autorisa à pénétrer et à s'installer dans l'immeuble en partie en ruines. "Puis en trois jours, les gens sont arrivés et c'était plein ", raconte une des trois fondatrices du lieu. D'autres réfugiés palestiniens venus de Chatila, puis d'autres en provenance d'autres camps de Beyrouth et d'autres lieux de la ville, les ont rejointes.

Aujourd'hui contrôlé par deux familles palestiniennes qui ont beaucoup investi dans la transformation de l'immeuble, Gaza Hospital est la résidence de familles palestiniennes et libano-palestiniennes, de familles syriennes anciennement installées et, plus récemment, de migrants travailleurs et de nombreux réfugiés syriens, mais aussi de migrants égyptiens, soudanais, et enfin de migrantes bangladaises plus récemment arrivées dans Gaza Hospital. Ces dernières louent des chambres en sous-sol, qui ont été construites par l'un des fils de l'une des deux principales familles du squat. On comptait au total, fin 2012, 127 logements de tailles variables (d'une ou deux pièces le plus souvent, à quelques très rares appartements de trois ou quatre pièces), et environ 500 habitants.

De huit étages lors de son ouverture, le squat en compte maintenant dix, et un onzième est en construction. Personne n'a de titre de propriété, mais on distingue sans trop de conflit qui est propriétaire, qui est hébergé gratuitement, et qui est locataire. Pour certains, peu nombreux, le squat est devenu une ressource objet d'investissement (constructions) et de profit (locations), même si l'impression d'ensemble est celle d'une "favela verticale ", extrêmement précaire sur le plan de l'hygiène, de l'alimentation en eau et électricité, du traitement des ordures et des eaux usés. Une ONG norvégienne, Norwegian Refugee Council (NRC), soutenue par l'Agence européenne pour l'action humanitaire ECHO, est intervenue en 2008 pour améliorer l'assainissement de l'immeuble.

Ce sont les moins légitimes et les plus fragiles des habitants du camp de Chatila qui sont restés dans le squat de Gaza Hospital, puisqu'ils n'ont pas pu justifier d'un droit à une réinstallation dans le camp au moment de sa reconstruction sous l'égide de I'UNRWA, l'Agence des Nations unies pour les réfugiés palestiniens, après la fin de la " guerre des camps ". Illégitimes, soit parce qu'ils habitaient dans les zones non officielles de Chatila, en bordure des limites du camp, soit parce qu'ils étaient hébergés ou sous-locataires sans titre dans le camp lui-même. Sous bien des aspects donc, Gaza Hospital est une extension du camp de Chatila. C'est à ce titre d'ailleurs que I'ONG NRC est intervenue, à partir de son bureau situé dans le camp de Chatila. Les liens familiaux et amicaux restent forts avec les habitants de Chatila. Au quotidien, les quelques centaines de mètres qui séparent l'un et l'autre lieux sont vite et régulièrement parcourus. Mais le squat ne relève pas de l'exceptionnalité juridique et politique du camp, il se trouve sur un espace entièrement libanais et beyrouthin. Ainsi, à l'illégitimité

7 Sur I'histoire politique et urbaine du camp de Chatila, voir Abou-Zaki (2014). 
vis-à-vis de Chatila, les habitants de Gaza Hospital voient s'ajouter l'illégalité juridique de leur installation dans le quartier de Sabra - à moins que ce soit simplement un vide juridique sur le statut et le devenir des biens de l'autorité palestinienne après son départ de Beyrouth. S'ajoute aussi une mauvaise réputation liée à l'insalubrité du lieu ou aux rumeurs de trafics d'armes - comme si une " contagion " palestinienne était sortie des camps vers la ville en passant par Gaza Hospital.

Comment l'établissement et la pérennité de ce lieu ont-ils été possibles ? Comment s'est-il transformé, stabilisé, jusqu'à devenir, vingt-six ans après son ouverture, un lieu de présence urbaine durable pour plusieurs générations de réfugiés et migrants en situation précaire, avec une inscription possible (même si elle est marginale) dans la ville ? En tant que lieu contemporain, Gaza Hospital est l'aboutissement de plusieurs histoires. Une histoire palestinienne bien sûr, comme on l'a brièvement mentionnée. Mais aussi une histoire des conflits et des déplacements dans la région, dont le dernier épisode, syrien, a des effets considérables, sur les plans économique et social, sur la vie du squat. De nombreux réfugiés syriens (potentiels nouveaux locataires) viennent en effet s'ajouter aux familles et travailleurs syriens présents de plus longue date. C'est une histoire également de travailleurs migrants venant d'un périmètre géographique plus large - des ouvriers du bâtiment et des employées de maison essentiellement et dont les mobilités et les conditions de vie précaires (mais parfois de courte durée) à Beyrouth les conduisent vers le squat comme une des possibilités d'accès à la ville : travailleurs syriens, égyptiens, soudanais du Nord et du Sud, migrantes sri-lankaises, bangladaises, éthiopiennes. Une histoire urbaine enfin, celle de la ville de Beyrouth, qui fait de Sabra une région morale aujourd'hui différente du "groupement palestinien " qu'elle a été dans les années 19601980. Sabra, que les commentaires beyrouthins qualifient de "zone de misère cosmopolite " et que Gaza Hospital incarne à sa façon de la manière la plus complète. Regroupant plusieurs générations et plusieurs vagues de migrants et de réfugiés, le squat est le lieu d'une altérité renouvelée dans la ville en même temps qu'un lieu de la mobilité. Même stabilisés (pour certains, les plus âgés, depuis vingt à vingt-sept ans), celles et ceux qui y vivent s'imaginent en transit. Les réfugiés palestiniens et syriens attendent (ou feignent d'attendre) un retour sur les terres qu'ils ont dû fuir, et les migrants voient leur passage à Gaza Hospital comme une étape dans un cycle plus long de mobilité.

\section{Conclusion : la frontière, le dehors et le sujet}

Les situations qui viennent d'être brièvement décrites incarnent un " dehors ", lointain ou proche : le dehors lointain est celui d'où viennent les occupants d'une part, et le dehors proche se localise dans une extraterritorialité, subie ou voulue, à la limite de l'ordre urbain, d'autre part. Car ce dont il s'agit n'est pas seulement un dehors des frontières administratives de l'État-nation (même si la figure du migrant international est généralement associée à celle de l'invasion suivie de l'occupation), c'est tout autant un dehors de l'ordre urbain. Un élément nouveau, imprévu dans l'agencement urbanistique, arrive par un lieu qui fait fonction de frontière. $C^{\prime}$ est ce qui fait de la frontière le lieu du sujet, politique ou urbain, manifestation physique d'un dérangement et d'un désaccord à propos du partage du lieu établi, et en particulier de la ville. 
Logiquement, la place de ce sujet est à la limite extérieure du périmètre administratif ou urbanistique de la ville, comme le sont les campements haoussas en Afrique de l'Ouest, présentés plus haut : le zongo (campement) se définit par distinction du birni (la ville) : la relation est essentielle à l'existence de l'un et de l'autre.

Si les campements d'étrangers se situent dans les " marges " de la ville (le terme de " marges " n'ayant a priori aucune connotation sociale, mais bien anthropologique et spatiale), ils peuvent être reliés à d'autres réalités sociales et spatiales, intégrés dans des réseaux de lieux comme on l'a vu avec le cas du campement de Patras, qui se définit non seulement par sa localisation sur une marge de la ville et proche d'une frontière nationale, mais aussi par sa place sur la route transnationale des migrants ${ }^{8}$. Dans le cas de Patras, le campement urbain s'est superposé au campement frontalier. Ainsi, " je me suis réfugié là " est la phrase politique du migrant afghan établi dans un campement à Patras (Grèce) comme à Calais (Nord de la France), à la limite de la ville et à l'entrée de son port frontalier. Puis cela devient un lieu où le migrant en marche reste, au prix d'une certaine insistance et obstination, mais aussi grâce à un compromis avec une autorité publique urbaine ou nationale. La situation de frontière devient progressivement un paysage qui s'installe dans la durée et dans l'espace de la ville à sa limite, au prix d'un compromis instable entre des personnes en mouvement d'une part, et d'autre part la société urbaine et le pouvoir urbain que ces personnes en déplacement rencontrent. Cette instabilité politique favorise le "flottement " et l'incertitude à propos des limites et du devenir de ces lieux de la limite. Elles obligent à penser la ville et sa dynamique dans un cadre plus vaste, celui d'une tension aujourd'hui de plus en plus conflictuelle entre mobilité et immobilité.

\section{Références bibliographiques}

Abou-Zaki Hala (2014) Chatila (Liban). Histoire et devenir d'un camp de réfugiés palestiniens, in Michel Agier Dir., Un monde de camps, Paris, La Découverte, pp. 35-46.

Agier Michel (Dir.) (2014a) Un monde de camps, Paris, La Découverte, 350 p.

Agier Michel (2014b) Toute la rugosité du monde, in Jean Birnbaum Dir., Repousser les frontières?, Paris, Folio Gallimard, pp. 87-101.

Agier Michel (2013) La condition cosmopolite, Paris, La Découverte, 240 p.

Agier Michel (1999) L'invention de la ville. Banlieues, townships, invasions et favelas, Paris, Éditions des Archives contemporaines, $176 \mathrm{p}$.

Agier Michel et Prestianni Sara (2011) " Je me suis réfugié là ! " Bords de routes en exil, Paris, Éditions Donner lieu, 126 p.

Appadurai Arjun (2005) Après le colonialisme. Les conséquences culturelles de la globalisation [traduit de I'anglais par Françoise Bouillot], Paris, Payot, 326 p.

8 On se trouve dans une situation proche de celle du " cosmopolitisme migratoire " évoqué par Tarrius (2012), mais je ne pense pas que I'hypothèse de la "diaspora " et son " territoire " s'impose pour comprendre ces réseaux de lieux cosmopolites. 
Augé Marc (1992) Non-lieux. Introduction à une anthropologie de la surmodernité, Paris, Seuil, $150 \mathrm{p}$.

Bauman Zygmunt (1999) Le coût humain de la mondialisation, Paris, Hachette Littérature, 204 p.

Bayart Jean-François (2004) Le gouvernement du monde. Une critique politique de la globalisation, Paris, Fayard, 454 p.

Burawoy Michael (Dir.) (2000) Global Ethnography: Forces, Connections, and Imaginations in a Postmodern World, Berkeley, University of California Press, $410 \mathrm{p}$.

Cessou Jean-Marie (1928) Les communautés islamiques, Écho des Missions africaines de Lyon, 1, pp. 29-32 et 2, pp. 55-57.

Clochard Olivier (Dir.) (2012) Atlas des migrants en Europe, Paris, Armand Colin, $144 \mathrm{p}$.

Daas Veena and Poole Deborah (2004) Anthropology in the Margins of the State, Santa Fé, School of Adavanced Research Press, 354 p.

Doraï Kamel et Puig Nicolas (Dir.) (2012) L'urbanité des, marges. Migrants et réfugiés dans les villes du Proche-Orient, Paris/Beyrouth, Éditions Ifpo/Téraèdre, $240 \mathrm{p}$.

Gupta Akhil and Ferguson James (Dir.) (1997) Culture, Power, Place: Explorations in Critical Anthropology, Durham, Duke University Press, 376 p.

Schütz Alfred (2010) L'Étranger. Un essai de psychologie sociale (1944), suivi de L'Homme qui rentre au pays (1945), Paris, Allia, $80 \mathrm{p}$.

Simmel Georg, (1984) Digressions sur l'étranger, in Yves Grafmeyer et Isaac Joseph Dir., L'École de Chicago. Naissance de l'écologie urbaine, Paris, Aubier, pp. 53-59.

Spire Amandine (2011) L'Étranger et la ville en Afrique de l'Ouest. Lomé au regard d'Accra, Paris, Karthala, $378 \mathrm{p}$.

Tarrius Alain (2012) Des transmigrants en France. Un cosmopolitisme migratoire original, Multitudes, 49, pp. 42-52. 


\section{Michel Agier}

\section{Parcours dans un paysage flottant de frontières}

Une bonne part du malaise contemporain vient du fait qu'avec la mondialisation humaine, avec le développement des circulations dans des périmètres toujours plus vastes, vers des points plus éloignés et des mobilités plus fréquentes, les situations de frontière sont plus nombreuses, plus complexes, alors que les personnes engagées dans ces situations y sont peu ou pas préparées. Faute de savoir identifier ces situations, nous risquons tous ensemble de perdre de vue l'importance voire la centralité qu'elles occupent dans la "fabrication " du monde. La description de trois lieux urbains de frontière (un quartier d'étrangers à Lomé au Togo, un campement de migrants à Patras en Grèce, un squat de réfugiés et migrants à Beyrouth au Liban) nous permet d'entrer dans ce paysage de frontières, incertain et inachevé.

\section{A Journey in a Floating Borderland}

A good part of the contemporary discontent comes from the fact that, with the human globalization, the development of circulations in larger and larger perimeters going to more distant locations, borders' situations are more numerous, more complex, although people engaged in these situations are sparsely or not at all prepared. Not knowing how to identify such situations, we risk all together losing sight the importance or even the centrality they occupy in the "making" of the world. The description of three border' urban places (a foreigners' neighborhood in Lomé, Togo, a migrants' encampment in Patras, Greece, a migrants and refugees' squat in Beirut, Lebanon) will allow us to enter into this uncertain and unfinished borderland.

\section{Un viaje en un paisaje flotante de fronteras}

Buena parte del malestar contemporáneo viene del hecho que, con la mundialización humana, con el desarrollo de circulaciones en perímetros siempre más grandes, para puntos más distantes y con movilidades más frecuentes, la situaciones de frontera se vuelven más numerosas y complejas, siendo que las personas engajadas en estas situaciones no son preparadas o poco para vivirlas. No sabiendo identificar tales situaciones, todos corremos el riesgo de perder de vista la importancia o hasta la centralidad que ellas ocupan en la "fabrica" del mundo. La descripción de tres lugares urbanos de frontera (un barrio de extranjeros en Lomé, Togo, un acampamiento de migrantes en Patras, Grecia, y un "squat" de refugiados y migrantes en Beirut, Líbano) nos permite entrar en este paisaje, incierto e inacabado, de frontera. 